\title{
Comparative Study between Analgesic Effects of Intrathecal Administration of Midazolam Versus Tramadol with Bupivacaine for Infra-Umbilical Surgeries
}

Mohamed Abdel Raheem Mohamed, Hitham Mohamed Ali, Fawzy Abbas Badawy*

Department of Anesthesia and Intensive Care, Faculty of Medicine, Sohag University, Sohag, Egypt.

*Corresponding author: Fawzy Abbas Badawy, Mobile: (+20) 01004862474, E-Mail: fabadawy@ gmail.com

\begin{abstract}
Background: The administration of bupivacaine intrathecally as a regional anesthesia is commonly used to provide anesthesia for lower abdominal surgeries. The motor blockage of bupivacaine provides adequate blockage, which allows the surgeons to do their work comfortably and also provides effective postoperative analgesia in early postoperative period. However, adding adjuvants to the local anesthetics to prolong the period of postoperative analgesia after fading of the spinal anesthesia was tried in many studies. These supplements as regard to postoperative pain relief are beneficial as they decrease or abolish the need for the administration of analgesics and their associated complications.

Objective: To compare the analgesic effect of intrathecal midazolam versus intrathecal tramadol when added to bupivacaine during spinal anesthesia after infra-umbilical surgeries.

Patients and methods: In our study we included 80 patients scheduled for elective surgeries in the lower abdomen and lower limbs (infra-umbilical surgeries) under spinal anesthesia. They were randomly divided to one of two groups. Group $\mathrm{M}$ received $10 \mathrm{mg}$ of hyperbaric bupivacaine $+2 \mathrm{mg}$ midazolam, and group $\mathrm{T}$ received $10 \mathrm{mg}$ of bupivacaine $+20 \mathrm{mg}$ of tramadol (total is $3 \mathrm{cc}$ in both groups). The comparison was done by using the visual analogue scale (VAS) for assessing postoperative pain score and recording the duration of postoperative analgesia.

Results: The postoperative analgesia duration was significantly higher in the tramadol group than midazolam group $(\mathrm{P}<0.05)$.

Conclusion: Addition of $20 \mathrm{mg}$ tramadol to bupivacaine during spinal anesthesia provides longer duration of analgesia when compared to intrathecal midazolam $2.0 \mathrm{mg}$.
\end{abstract}

Keywords: Midazolam, Tramadol, Bupivacaine, Infra-umbilical surgeries.

\section{INTRODUCTION}

Spinal anesthesia is the most commonly used technique for lower abdominal surgeries as it is easy to administer and of less $\operatorname{cost}^{(\mathbf{1})}$. It also has the advantage that profound nerve block can be produced in a large part of the body by the relatively simple injection of a small amount of local anesthetic (2), and avoids the risks of general anesthesia such as aspiration of gastric contents and difficulty with airway management ${ }^{(3)}$. Bupivacaine is suitable for procedures lasting up to 90-120 minutes ${ }^{(4)}$, and is the most commonly used local anesthetic agent for spinal anesthesia. But it doesn't provide prolonged postoperative analgesia. So supplements were suggested to prolong postoperative analgesia. Intrathecal opioid administration has been demonstrated to provide

the nervous system, including the spinal

cord. Midazolam is a water-soluble benzodiazepine with sedative, amnesic, anxiolytic, muscle relaxant, and anticonvulsant properties ${ }^{(11,12)}$.

Midazolam can be given by intrathecal or epidural injection to provide postoperative analgesia, and also produce an anti-nociceptive effect. This may be gamma-aminobutyric acid (GABA) mediated. The gamma-aminobutyric acid has been shown to have analgesic properties. There are many uses for midazolam during the perioperative period including premedication, anesthesia induction, and maintenance of sedation for diagnostic and therapeutic procedures ${ }^{(\mathbf{1 3})}$. Our study effective postoperative analgesia after a variety of surgical procedures, but with increased risk for respiratory depression ${ }^{(\mathbf{5})}$.

But tramadol is a centrally acting analgesic that has minimal respiratory depressant effects ${ }^{(6,7)}$, as its 6000-fold decreased affinity for mu-receptors compared with morphine ${ }^{\mathbf{8 , 9 )}}$. It also inhibits serotonin and norepinephrine reuptake in the spinal cord and has no reported neural toxicity ${ }^{(\mathbf{1 0})}$. So tramadol has the potential to provide effective postoperative analgesia with no risk of respiratory depression after intrathecal administration.

throughout

Benzodiazepine receptors are present aimed to compare the analgesic effect of intrathecal midazolam versus intrathecal tramadol when added to bupivacaine during spinal anesthesia after infraumbilical surgeries.

\section{MATERIALS AND METHODS}

\section{Ethical approval:}

This study was performed after approval of Ethical Committee of Faculty of Medicine, Sohag University. Eighty patients aged between 18-70 years with ASA I-II who were scheduled for elective infra-umbilical surgeries under spinal anesthesia were enrolled in our 
study after obtaining informed consent from every patient.

\section{Inclusion criteria:}

1- ASA physical status I and II

2- Aged 18-70

3- Both gender

4- Patient scheduled for elective lower abdomen and lower limbs surgeries.

\section{Exclusion criteria:}

Patients with a history of

1- Opium addiction, sedative drugs consumption.

2- Contraindication for spinal anesthesia, as sepsis, severe coagulopathy and severe heart disease.

3- Patient with long history of steroid use.

4- Patient unable to communicate making postoperative assessment difficult.

Routine investigations were done for all patients (CBC, coagulation profile, liver function tests, kidney function tests, blood glucose level). Patients did not receive premedication.

On arrival of patients into the operating room; patients were monitored for ECG, pulse oximetry $\left(\mathrm{SPO}_{2}\right)$ and noninvasive blood pressure (NIBP). Patients were cannulated and $10-15 \mathrm{ml} / \mathrm{kg}$ of isotonic saline was infused as preload.

\section{Patients were divided into two equal groups:}

-(Group M) 40 patients received $2.0 \mathrm{mg}$ midazolam added to $2.5 \mathrm{ml}$ volume of $0.5 \%$ hyperbaric bupivacaine (3 ml total).

-(Group T) 40 patients received $20 \mathrm{mg}$ tramadol added to $2.5 \mathrm{ml}$ volume of $0.5 \%$ hyperbaric bupivacaine $(3 \mathrm{ml}$ total).

Patient were observed for pulse, oxygen saturation, systolic, diastolic and mean blood pressure. We recorded the baseline readings. Then, while patients were in a sitting position, spinal anesthesia was done under sterile conditions with a 25-gauge spinal needle between L4-L5 lumbar regions using $12.5 \mathrm{mg}$ hyperbaric bupivacaine $0.5 \%$ plus $0.5 \mathrm{ml}$ of the adjuvant, totally $3 \mathrm{cc}$ was injected. The sensory blockage was assessed by the loss of sensation in response to pinprick. The time to onset of the sensory block, and time to achieve maximum sensory block were recorded. The sensory block was assessed by pinprick to skin every 2 minutes till the level stabilized for three consecutive tests. Regression time, to reach sensory level two segments from the highest level and regression time to reach sensory level up to L5-S1 was recorded. A dermatomal sensory loss $\mathrm{T}_{7}$ was considered satisfactory. Intensity of the motor blockade was assessed by the Bromage scale ${ }^{(\mathbf{1 4})}$ (As a block extends cephalad, there is progressive impairment of motor as well as sensory function, where (0) means no motor block, (1) means inability to raise extended leg; able to move knees and feet, (2) means inability to raise extended leg and move knee; able to move feet, and (3) means complete block of motor limb). Complete inability to straight leg raise (Bromage grade 3). The duration of surgery for each case was recorded. Duration of analgesia was measured as the time from induction of block to first patient request for analgesia.

The patients were explained about the spinal anesthesia technique and educated regarding the Visual Analogue Scale (VAS), advocated by Revill and Robinson in 1976 . The VAS consists of a $10 \mathrm{~cm}$ line at one end by the label "no pain" and at the other end with "the worst pain imaginable" (Figure 1) ${ }^{(\mathbf{1 5})}$.

Standard monitoring was carried out in the form of ECG, pulse oximetry, heart rate, and noninvasive arterial blood pressure was recorded every 5 minutes intraoperatively. No sedation or analgesics were given to the patients during surgery. Postoperatively, patients were examined every 30 minutes for 12 hours to evaluate the duration and quality of postoperative pain relief.

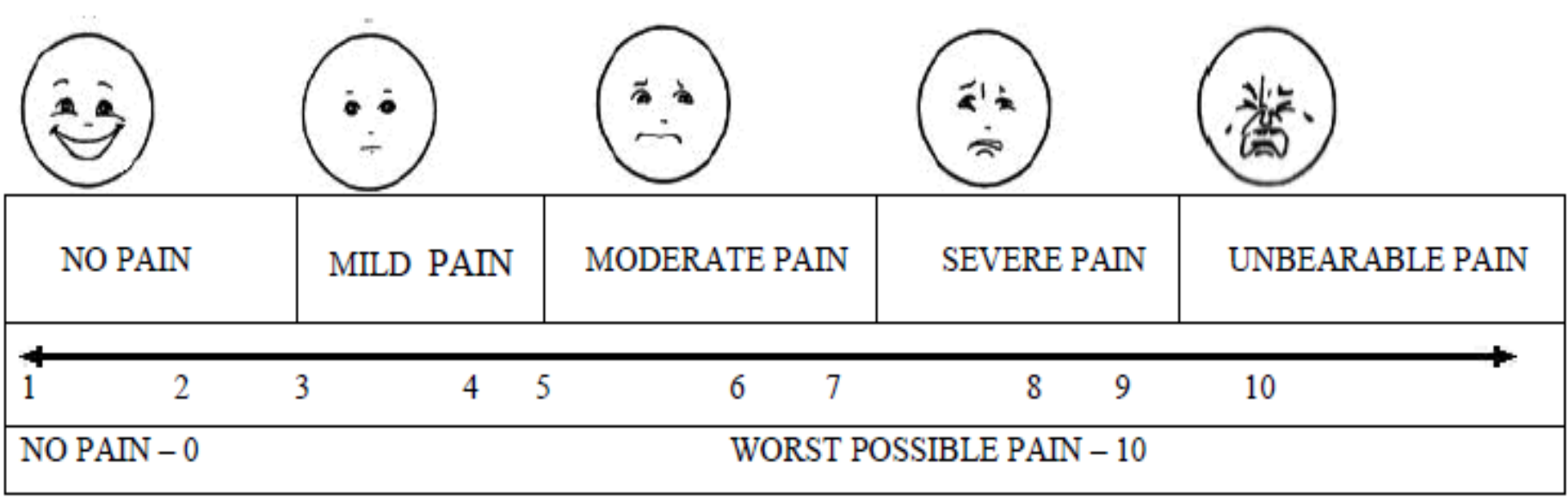

Figure (1): Visual analogue scale ${ }^{(15)}$. 
Supplemental analgesia was given when the result of the VAS was greater than 4. The time of supplemental analgesia administration was noted.

Following recovery, time to regression to level $\mathrm{L}_{5}$ $\mathrm{S}_{1}$ was recorded. The motor power was assessed by Bromage scale in minutes.

Side effects were recorded; mainly hypotension, bradycardia, sedation, nausea and vomiting, pruritus, shivering, urinary retention, and respiratory depression. Hypotension is defined as reduction of systolic blood pressure less than $90 \mathrm{mmHg}$ or $<20 \%$ of baseline and was treated with increased rate of intravenous fluids and if needed injection of intravenous ephedrine $5 \mathrm{mg}$ given in increments.

\section{Statistical analysis}

In this study, we analyzed the statistical significance of the differences between group $\mathrm{T}$ (tramadol) and group M (Midazolam) by Statistical Package for the Social Sciences (SPSS) version 16. Quantitative data were expressed as mean \pm SD and were compared by unpaired Student's $t$ test. Qualitative data were expressed as frequency and percentage and were compared by $\mathrm{Chi}^{2}$ test. $\mathrm{P}$ value $<0.05$ was considered statistically significant.

\section{RESULTS}

All the eighty patients completed the study with no statistically significance as regard the demographic data (table 1). The average duration of surgery in both groups was nearly equal.

Table (1): Demographic data

\begin{tabular}{|l|c|c|c|}
\hline & $\begin{array}{c}\text { Group M (mean } \pm \text { SD or } \\
\text { frequency) } \\
\mathbf{N = 4 0}\end{array}$ & $\begin{array}{c}\text { Group T (mean } \pm \text { SD or } \\
\text { frequency) } \\
\mathbf{N = 4 0}\end{array}$ & P value \\
\hline Age (years) & $49.56 \pm 17$ & $46.81 \pm 16$ & $>0.05$ \\
\hline Height $(\mathrm{cm})$ & $158.47 \pm 12.6$ & $155.96 \pm 15.8$ & $>0.05$ \\
\hline Weight $(\mathrm{kg})$ & $78.53 \pm 15.32$ & $74.98 \pm 16.78$ & $>0.05$ \\
\hline Sex (male/female) & $24 / 16$ & $19 / 21$ & $>0.05$ \\
\hline Duration of surgery $(\min )$ & $95.67 \pm 20.42$ & $92.52 \pm 14.38$ & $>0.05$ \\
\hline
\end{tabular}

As regard to time to onset of sensory block, there was statistically significant difference in mean time to achieve highest level of block (Table 2).

Table (2): Sensory and motor characteristics

\begin{tabular}{|l|c|c|c|}
\hline & $\begin{array}{c}\text { Group M } \\
\mathbf{N = 4 0}\end{array}$ & $\begin{array}{c}\text { Group T } \\
\mathbf{N = 4 0}\end{array}$ & P value \\
\hline Onset of sensory block (min) & $2.85 \pm 0.21$ & $3.15 \pm 0.17$ & $<0.001$ \\
\hline $\begin{array}{l}\text { Time to highest sensory block } \\
\text { (min) }\end{array}$ & $4.23 \pm 1.48$ & $7.69 \pm 1.24$ & $>0138^{*}$ \\
\hline $\begin{array}{l}\text { Time of Sensory regression to } \\
\text { L5-S1 (min). }\end{array}$ & $260.48 \pm 43.39$ & $273.82 \pm 3.658$ & $>0.05$ \\
\hline Time to reach Bromage scale 3 & $11.87 \pm 1.89$ & $12.45 \pm 2.13$ & $>0.05$ \\
\hline Time to reach Bromage scale 0 & $152.52 \pm 4.43$ & $155.20 \pm 4.85$ & 0.012 \\
\hline
\end{tabular}

Values: mean \pm SD. $*$ Statistically significant.

The VAS scores were comparable between both groups during the first 4 hours of immediate postoperative period, but after 4 hours, the VAS score was statistically significant difference between the two groups. In midazolam group, $32.5 \%$ of patients did not require any analgesia within 8 hours (Table 3), while none in tramadol group (Table 4).

Table (3): Visual analogue score for group M

\begin{tabular}{|c|c|c|c|}
\hline V A S & $\begin{array}{c}\mathbf{0 - 4} \text { hours } \\
\mathbf{n}(\boldsymbol{\%})\end{array}$ & $\begin{array}{c}\mathbf{4 - 8} \text { hours } \\
\mathbf{n}(\%)\end{array}$ & $\begin{array}{c}\mathbf{8 - 1 2} \text { hours } \\
\text { n (\%) }\end{array}$ \\
\hline $1-2$ & $37(92.5 \%)$ & $4(10 \%)$ & --- \\
\hline $3-4$ & $3(7.5 \%)$ & $14(35 \%)$ & --- \\
\hline $5-6$ & --- & $13(32.5 \%)$ & $12(30 \%)$ \\
\hline $7-8$ & --- & $20(50 \%)$ & --- \\
\hline
\end{tabular}

$\mathrm{n}=$ number of patients who did not require analgesia. VAS $=$ visual analogue score. 
Table (4): Visual analogue score for group T

\begin{tabular}{|c|c|c|c|}
\hline V A S & $\begin{array}{c}\mathbf{0 - 4} \text { hours } \\
\mathbf{n}(\boldsymbol{\%})\end{array}$ & $\begin{array}{c}\mathbf{4 - 8} \text { hours } \\
\mathbf{n}(\boldsymbol{\%})\end{array}$ & $\begin{array}{c}\mathbf{8 - 1 2} \text { hours } \\
\text { n (\%) }\end{array}$ \\
\hline $1-2$ & $38(95 \%)$ & $4(10 \%)$ & $12(30 \%)$ \\
\hline $3-4$ & $2(2.5 \%)$ & $32(80 \%)$ & $5(12.5 \%)$ \\
\hline $5-6$ & --- & --- & $4(10 \%)$ \\
\hline $7-8$ & --- & $3(7.5 \%)$ & $17(42.5 \%)$ \\
\hline
\end{tabular}

$\mathrm{n}=$ number of patients who did not require analgesia. VAS $=$ visual analogue score.

Postoperative analgesia was given to all patients in midazolam group at a mean duration of $486.76 \pm 60.6$ minutes after induction of anesthesia. Only 3 patients in tramadol group demanded postoperative analgesia within this period (when VAS > 4). Postoperative analgesia was supplemented in all patients in tramadol group at a mean duration of $689.68 \pm 62.2$ minutes postoperatively. The difference in mean postoperative supplemental analgesic time between the 2 groups was statistically significant (Figure 2).

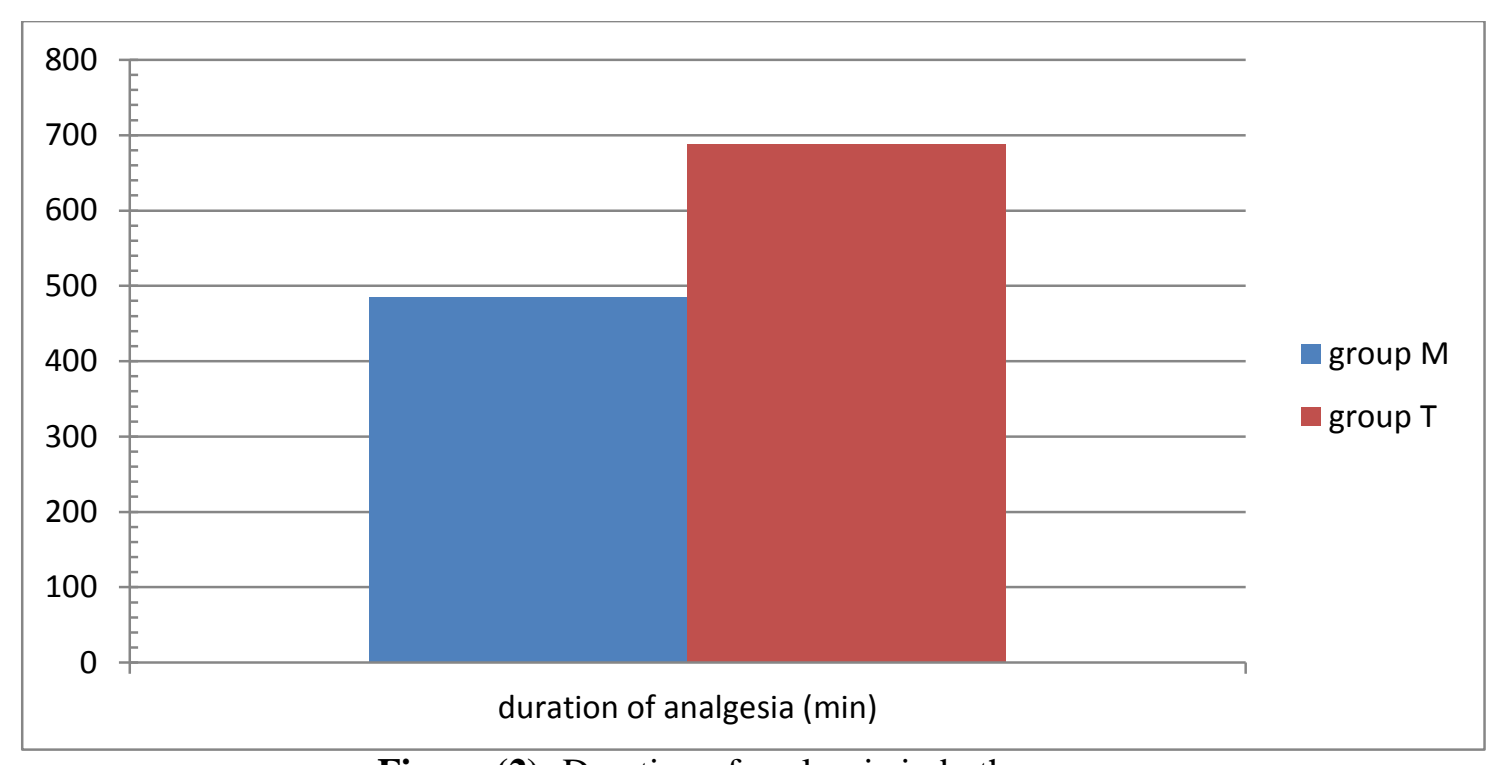

Figure (2): Duration of analgesia in both groups.

As regard to heart rate, systolic, and diastolic blood pressures changes between the two groups; there was no statistically significant difference (Table 5) in between both groups.

Table (5): Changes in heart rate, systolic, and diastolic pressures

\begin{tabular}{|l|c|c|c|}
\hline & Group M & Group T & P value \\
\hline Heart rate b/m & $90.4 \pm 7.32$ & $92.6 \pm 6.81$ & $>0.05$ \\
\hline $\begin{array}{l}\text { Systolic blood pressure } \\
(\mathrm{mm} \mathrm{Hg})\end{array}$ & $116.73 \pm 8.31$ & $121.52 \pm 6.73$ & $<0.01$ \\
\hline $\begin{array}{l}\text { Diastolic blood pressure } \\
(\mathrm{mmHg})\end{array}$ & $78.46 \pm 5.24$ & $77.32 \pm 4.89$ & $>0.05$ \\
\hline
\end{tabular}

Values $=$ mean \pm standard deviation (SD).

As regard to incidence of complication or side effects, there was no statistically significant differences in between groups (Table 6), except the occurrence of shivering which was significantly lower in tramadol group. Also, there was higher incidence of vomiting and sedation in tramadol group with statistically significant difference.

Table (6): Side effects

\begin{tabular}{|l|c|c|c|}
\hline & Group M (n) & Group T (n) & P value \\
\hline Sedation & 6 & 10 & $>0.05$ \\
\hline Vomiting & 5 & 11 & $>0.05$ \\
\hline Resp. depression & 0 & 0 & $====$ \\
\hline Shivering & 7 & 2 & $>0.05$ \\
\hline Pruritus & 0 & 0 & $====$ \\
\hline Urinary retention & 2 & 3 & $>0.05$ \\
\hline
\end{tabular}

$\mathrm{n}=$ number of patients. $*$ Statistically significant difference. 


\section{DISCUSSION}

Performing spinal anesthesia with the addition of any adjuvant is well established nowadays, as it reduces the severity of postoperative pain and prolongs analgesia even after recovery from sensory and motor blockades. Prolongation of the duration of (after the end of bupivacaine action) postoperative pain relief protected our patients from several complications as atelectasis, urinary retention, prolonged hospital stay and increased healthcare costs ${ }^{(16)}$.

Our finding in the present study showed that the addition of midazolam or tramadol to bupivacaine $0.5 \%$ to control postoperative pain in spinal anesthesia provided the anesthesiologists alternative medications with fewer side effects to increase the analgesia duration and decrease pain intensity, but shorter duration than the cornerstone (morphine) of postoperative analgesia. Morphine exerts its analgesic effects through $\mu$ receptors at spinal and supra-spinal level. Tramadol acts as weak $\mu$-receptor agonist and to a lesser extent delta

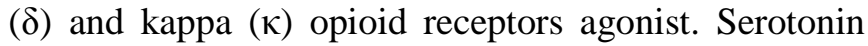
reuptake inhibition and norepinephrine reuptake inhibition also contributes to its analgesic properties ${ }^{(16)}$.

The administration of the benzodiazepine antagonist flumazenil and the GABA-A antagonist bicuculline has been reported to reverse the analgesic effect of intrathecal midazolam, suggesting that the antinociceptive actions are mediated via the benzodiazepine and GABA-A receptor complexes, which are abundantly present in lamina II of the dorsal horn ganglia of the spinal cord $^{(17)}$. Intrathecal midazolam probably also causes the release of an endogenous opioid acting on the spinal delta receptor as naltrindole, which is a delta selective opioid antagonist, and which suppresses the analgesic effect of intrathecal midazolam ${ }^{(\mathbf{1 8 )}}$.

Our study showed that the addition of midazolam or tramadol to intrathecal bupivacaine prolongs the duration of postoperative analgesia. The time to first analgesic demand was $689.68 \pm 62.2$ minutes in group $\mathrm{T}$ compared to $486.76 \pm 60.6$ minutes in group $M$. So there is statistically significant prolongation of postoperative analgesia with tramadol when compared with midazolam in patients undergoing infraumbilical surgeries.

Also, pain intensity scoring by VAS at 4,8 and 12 hours after surgery was significantly lower in the tramadol group compared to the midazolam group.

There was no significant difference between duration of surgery in both the groups. It was $95.67 \pm$ 20.42 in midazolam group and $92.52 \pm 14.38$ in tramadol group. Also, our study showed that the addition of 2.0 $\mathrm{mg}$ midazolam or $20 \mathrm{mg}$ tramadol to $10 \mathrm{mg}$ of spinal bupivacaine did not show significant difference in the time for onset of sensory blockade. In both groups highest level attained was T7. Time to achieve T7 level was more rapid with group $\mathrm{M}$ than group $\mathrm{T}$ and the difference was significant statistically $(\mathrm{P}<0.05)$.

Batra et al. ${ }^{(19)}$, observed that the mean duration of time to recede to the $\mathrm{L}_{5}-\mathrm{S}_{1}$ sensory level was $267 \pm$ 67.38 minutes, and observed superior and prolonged postoperative analgesia (they only compared midazolam with saline) which had shorter duration than our study.

Similar studies have also suggested that intrathecal addition of midazolam or tramadol (but the addition was to lidocaine 5\%) during spinal anesthesia could effectively increase the duration of postoperative analgesia and reduce pain intensity. A study was done by Pazuki et al. ${ }^{(20)}$ compared intrathecal injection of tramadol versus midazolam as regard analgesia duration and postoperative shivering after cesarean section, and found that tramadol was more effective than midazolam and it increased analgesia duration and reduced postoperative shivering after cesarean section, which is in agreement with our study.

Also a study done by Sen $\boldsymbol{e t}$ al. ${ }^{(21)}$, evaluated the effects of midazolam $(2 \mathrm{mg}$ ) on postoperative pain control, vital signs of the mother and one- and fiveminute Apgar scores of infants in 40 women, and they found that intrathecal administration of midazolam could effectively control the pain after cesarean section, while exerting anti-nausea and tranquilizing effects.

Chakraborty et al. ${ }^{(22)}$, also found that $20 \mathrm{mg}$ of tramadol added to $15 \mathrm{mg}$ of bupivacaine effectively prolonged the duration of analgesia from $210 \pm 10.12$ min in bupivacaine saline group to $380 \pm 11.82 \mathrm{~min}$ in bupivacaine-tramadol group, which is in correlation with our results.

Other studies done with tramadol in regional anesthesia by Kumari et al. (23), Frikha et al. (24), Brijesh et al. (25) and Ozcengiz et al. ${ }^{(26)}$ demonstrated the efficacy of tramadol as an adjuvant in spinal epidural or caudal anesthesia in place of pure opioid agonists.

On the other hand, some studies proved no benefit of adding tramadol to spinal anesthesia as found by Alhashemi et al. ${ }^{(27)}$. He did not find any benefit in postoperative analgesia for TURP (transurethral resection of prostate) patients when intrathecal tramadol was used. Grace et al. ${ }^{(28)}$ and Wilder-smith et al. ${ }^{(29)}$ also did not find any benefit of intrathecal tramadol over bupivacaine alone.

\section{CONCLUSION}

We concluded according to our study that intrathecal tramadol provide very good and prolonged postoperative analgesia without significant side effects compared to intrathecal midazolam.

\section{REFERENCES}

1. Bani-Hashem N, Hassan-Nasab B, Pour E et al. (2011): Addition of intrathecal dexamethasone to 
bupivacaine for spinal anesthesia in orthopedic surgery. Saudi J Anaesth., 5:382-386.

2. Shashni S, Abhijit S, Gopal T (2014): Clinical effects of intrathecal midazolam versus intrathecal magnesium sulfate as adjuncts to hyperbaric bupivacaine: A comparative study. Indian Journal of Pain, 3: 175-181.

3. Fouad H, Ahmed A, Osman Y et al. (2016): Efficacy of preemptive dexamethasone added to bupivacaine in ultrasound guided transversus abdominus plain block for postoperative analgesia after inguinal herniorraphy. American Journal of Research Communication, 4(5): 2742.

4. Soave P, Conti G, Costa $\mathbf{R}$ et al. (2009): Magnesium anaesthesia. Curr Drug Targets, 10:734-43.

5. Jacobson L, Chabal C, Brody M (1988): A doseresponse study of intrathecal morphine: efficacy, duration, optimal dose, and side effects. Anesth Analg., 67: 1082-8.

6. Vickers M, O'Flaherty D, Szekely S et al. (1992): Tramadol: pain relief by an opioid without depression of respiration. Anaesthesia , 47: 291-6.

7. Tarkkila $P$, Tuominen $M$, Lindgren $L$ (1998): Comparison of respiratory effects of tramadol and pethidine. Eur J Anaesthesiol., 15: 64-8.

8. Raffa R, Friderichs E, Reimann W et al. (1992): Opioid and nonopioid components independently contribute to the mechanism of action of tramadol, an ‘atypical' opioid analgesic. J Pharmacol Exp Ther., 260: 275-85.

9. Scott L, Perry C (2000): Tramadol: a review of its use in perioperative pain. Drugs, 60: 139-76.

10. Tsai Y, Chang P, Jou I (2001): Direct tramadol application on sciatic nerve inhibits spinal somatosensory evoked potentials in rats. Anesth Analg., 92: 1547-51.

11. Kanto J, Aaltonen $L$, Aatimaa $L$ (1984): Pharmacokinetics and selective effects of midazolam in connection with caesarean section performance under epidural analgesia. Acta Anaesthesiologica Scandinavia, 28(1): 116-118.

12. Shafer A (1998): Complication of selection with midazolam in the intensive care unit and a comparison with the other selective regimens. Critical care Medicine, 26 (5): 947-952.

13. Bromage $P$ (1981): Practice of spinal narcotic analgesia - Basic consideration. Anaesthesia Analgesia , 60: 461.

14. Bromage $P$ (1965): A comparison of the hydrochloride and carbon dioxide salts of lidocaine and prilocaine in epidural analgesia. Acta Anaesthesiol Scand., 16:55-69.

15. Meyer H (2001): Practical Management of Pain Bonica's Management of Pain. JAMA., 286 (15): 1906-1907.

16. Duthie D (1998): Remifentanil and tramadol. British Journal of Anesthesia, 81: 51-7.
17. Goodchild C, Guo Z, Gent J (1996): Antinociception by intrathecal midazolam involves endogenous neurotransmitters acting at spinal cord delta opioid receptors. British Journal of Anaesthesia, 77: 758-763.

18. Nicol M, Holdcroft A (1992): Density of intrathecal agents. British Journal of Anaesthesia, 68: 60-63.

19. Batra Y, Jain K, Chari P (1999): Addition of intrathecal midazolam to bupivacaine produces better postoperative analgesia without prolonging recovery. Int $\mathrm{J}$ Clin Pharmacology Therapeutics, 37 (10): 519-23.

20. Pazuki S, Kamali A, Shahrokhi N et al. (2016): Comparison of the effects of intrathecal midazolam and tramadol with the conventional method of postoperative pain and shivering control after elective cesarean section. Biomed Pharmacol J., 9(3): 995-1003.

21. Sen A, Rudra A, Sarkar S et al. (2001): Intrathecal midazolam for postoperative pain relief in caesarean section delivery. J Indian Med Assoc., 99(12):683-6.

22. Chakraborty S, Chakrabarti J, Bhattacharya D (2008): Intrathecal tramadol added to bupivacaine as spinal anesthetic increases analgesic effect of the spinal blockade after major gynecological surgeries. Indian $\mathbf{J}$ Pharmacol., 40: 180-182.

23. Kumari P, Bali K, Chatrath V et al. (2015): Fentanyl versus tramadol with levobupivacaine for combined spinal-epidural analgesia in labor. Saudi J Anaesth., 9: 263- 67.

24. Frikha N, Massoudi K, Ouergh S (2008): Spinal tramadol for caesarean section. Obstet Anaesth., 8: 72-78.

25. Brijesh J, Sarasawat V (2001): Efficacy of intrathecal tramadol for post-operative pain relief in cases of L.S.C.S and major gynecological surgeries-A clinical evaluation. Indian J Anaesth., 45: 282-87.

26. Ozcengiz D, Gunduz M, Ozbek H et al. (2001): Comparison of caudal morphine and tramadol for postoperative pain control in children undergoing inguinal herniorrhaphy. Pediatric Anesthesia , 11: 459464.

27. Alhashemi J, Kaki A (2003): Effect of intrathecal tramadol administration on postoperative pain after transurethral resection of prostate. Br J Anaesth., 91: 53640.

28. Grace D, Fee J (1995): Ineffective analgesia after extradural tramadol hydrochloride in patients undergoing total knee replacement. Anesthesia , 50: 555-8.

29. Wilder-Smith C, Wilder-Smith O, Farschtschian M et al. (1998): Preoperative adjuvant epidural tramadol: the effect of different doses on postoperative analgesia and pain processing. Acta Anaesthesiol Scand., 42: 299-305. 